\title{
Structural identification of Egnatia Odos bridges based on ambient and earthquake induced vibrations
}

\author{
Evaggelos Ntotsios ${ }^{1}$, Christos Karakostas ${ }^{2 *}$, Vasilios Lekidis ${ }^{2}$, Panagiotis \\ Panetsos $^{3}$, Ioannis Nikolaou ${ }^{1}$, Costas Papadimitriou ${ }^{4}$
}

\begin{abstract}
The dynamic characteristics of two representative R/C bridges on Egnatia Odos motorway in Greece are estimated based on low amplitude ambient and earthquake-induced vibrations. The present work outlines the instrumentation details, algorithms for computing modal characteristics (modal frequencies, damping ratios and modeshapes), modal-based finite element model updating methods for estimating structural parameters, and numerical results for the modal and structural dynamic characteristics of the two bridges based on ambient and earthquake induced vibrations. Transverse, bending and longitudinal modes are reliably identified and stiffness-related properties of the piers, deck and elastomeric bearings of the finite element models of the two bridges are estimated. Results provide qualitative and quantitative information on the dynamic behavior of the bridge systems and their components under low-amplitude vibrations. Modeling assumptions are discussed based on the differences in the characteristics identified from ambient and earthquake vibration measurements. The sources of the differences observed between the identified modal and structural characteristics of the bridges and those predicted by finite element models used for design are investigated and properly justified.
\end{abstract}

Key words: Modal identification, model updating, ambient vibrations, earthquake excitations, bridges

\footnotetext{
${ }^{I}$ Graduate Student, Department of Mechanical and Industrial Engineering, University of Thessaly, Volos 38334, Greece (e-mail: entotsio@uth.gr, ionikola@uth.gr)

${ }^{2}$ Research Director, Institute of Engineering Seismology and Earthquake Engineering P.O. Box 53, GR 55102, Finikas, Thessaloniki, Greece (e-mail: christos@itsak.gr, lekidis@itsak.gr)

${ }^{3}$ Civil Engineer, PhD, Egnatia Odos S.A., Capital Maintenance Department, 6th km Thessaloniki-Thermi, P.O. Box 30, GR 57001 Thermi, Greece (e-mail: ppane@egnatia.gr)

${ }^{4}$ Professor, Department of Mechanical and Industrial Engineering, University of Thessaly, Volos 38334, Greece (e-mail: costasp@uth.gr)

* Correspondent Author
} 


\section{Introduction}

The evaluation of the actual dynamic characteristics of civil engineering structures through measurements of their dynamic response has been attracting an increasing research effort worldwide. Measured response data of bridges from ambient and earthquake-induced vibrations offer an opportunity to study quantitatively and qualitatively their dynamic behaviour within the resulting vibration levels. These vibration measurements can be processed for the estimation of the modal characteristics of the bridges, as well for the calibration of corresponding (finite element) models used to simulate their behaviour. The information for the identified modal models and the updated finite element models is useful for validating the assumptions used in model development or for improving modelling, analysis and design procedures. Also, such information is useful for structural health monitoring purposes.

The estimation of the modal characteristics requires the application of system identification methods that process output-only (ambient) as well as earthquakeinduced vibrations. Modal identification algorithms provide estimates of the modal frequencies, modal damping ratios and modeshapes at the measured DOFs using classically-damped or non-classically damped modal models. For the case of ambient vibrations, there are a number of methods and respective software developed either in time or frequency domain for the identification of modal properties. The methods, based on output measurements only, assume that the input can be well represented by a vector white noise process. Recent developments are reported in Peeters and De Roeck (1999, 2001) and Basseville at al. (2001) using time domain stochastic subspace identification methods, in Beck et al. (1994) using time domain least-squares methods based on correlation functions of the output time histories, in Verboten et al. (2002), Gauberghe (2004) and Brincker et al. (2001) using frequency domain least-squares methods based on full cross-power spectral densities (CPSD), and in Peeters and Van der Auweraer (2005) based on half spectra. Bayesian and maximum likelihood statistical methods have also been proposed, for example, in Katafygiotis and Yuen (2001), Guillaume et al. (1999) and Verboten (2002). For the case of earthquake-induced vibrations, modal identification methods have also been developed either in time (Beck 1978; Beck and Jennings 1980) or in frequency (McVerry 1980) domains, 
based on a minimization of the measure of fit between the time history or its Fourier transform of the acceleration responses estimated from the measurements and the corresponding ones predicted from a classically-damped modal model of the structure. Extensions for identifying non classically-damped modal models in the frequency domain has also been developed by Chaudhary et al. (2000). These methods have been applied to identify the modal characteristics of bridges (Werner et al. 1987; Chaudhary 2002) and buildings (Papageorgiou and Lin 1989) by processing input-output earthquake recordings.

Model updating algorithms based on the identified modal characteristics have also been developed (e.g. Mottershead and Friswell 1993; Bohle and Fritzen 2003; Teughels et al. 2003; Lam et al. 2004; Christodoulou and Papadimitriou 2007) to estimate structural parameters associated with a finite element model of a structure. This calibration procedure provides useful insight into the range of validity of the modelling assumptions and is used to improve our understanding of the dynamic behaviour of the structure and its components. Structural model parameter estimation methods based on the identified modal data are often formulated as weighted least-squares problems in which the optimal values of the structural parameters of a finite element model are obtained by minimizing a measure of the residuals between measured and finite element model predicted modal characteristics.

The proposed work develops and applies modal identification and model updating methodologies for estimating the dynamic modal and stiffness characteristics of two representative bridges on the Egnatia Odos motorway, using both ambient and earthquake induced vibration measurements. In the present paper, Section 2 gives an overview of the two bridges and the instrumentation procedure. Section 3 outlines the main points of the modal identification algorithms developed to estimate the modal characteristics from both ambient and earthquake induced vibration measurements. Section 4 outlines a weighted leastsquares residuals method for finite element model updating based on the identified modal characteristics. Results for the modal characteristics and the values of stiffness-related parameters of the piers, deck and bearings of the instrumented bridges are presented in Sections 5 and 6. Modeling assumptions are discussed based on the differences in the dynamic characteristics identified from ambient and earthquake vibration data. Moreover, modelling issues related to the 
structural behaviour of the bridges under low amplitude vibrations are investigated based on comparisons with analytical finite element models used for earthquake design of these bridges. The conclusions of this work are summarized in Section 7.

\section{Description of bridge systems and instrumentation}

Egnatia Motorway is a new, $670 \mathrm{~km}$ long highway, that transverses Northern Greece in an E-W direction. The two R/C bridges that were instrumented with special accelerometer arrays are the $9^{\text {th }}$ Ravine Bridge on the Veria - Polymylos section (Figure 1a) and the $2^{\text {nd }}$ Kavala Bypass Ravine Bridge (Figure 1b). Both bridges have two, almost identical, statically independent branches, one for each traffic direction, one of which was instrumented in each case.

The T-shaped $9^{\text {th }}$ Polymylos bridge is curved in plan and has a total length of $170 \mathrm{~m}$. The deck cross section is a box girder of height varying parabolically from $9 \mathrm{~m}$ at the central pier to $4 \mathrm{~m}$ at the two abutments. It is supported monolithically by a central pier (M1), of 35m height, which is founded on a massive rectangular $\mathrm{R} / \mathrm{C}$ rock socket at its basement and continues with two transverse flanges for the rest of its height. Each of the two 85m-long cantilever parts of the deck girder rests on each abutment through special elastomeric bearings that allow free sliding in the longitudinal direction (to accommodate thermal expansions/contractions), while functioning as normal elastomeric pads in the transverse (radial) direction.

The 2nd Kavala Bypass Ravine Bridge, with a total length of 170m, comprises four identical simply supported spans of $42.5 \mathrm{~m}$, each span built with four precast post-tensioned I-beams of $2.80 \mathrm{~m}$ height, supporting a $26 \mathrm{~cm}$ thick deck, which is continuous throughout the bridge length. The I-beams are supported through laminated elastomeric bearings on the two abutments and the three piers. The piers have a $4 \mathrm{~m} \times 4 \mathrm{~m}$ square cross-section with $40 \mathrm{~cm}$ wall thickness and heights of 30m (M1, M3) and 50m (M2). The four spans of the deck are interconnected through a 2-meter-long $20 \mathrm{~cm}$-thick continuity slab over the piers.

Two 12-channel Kinemetrics K2 ${ }^{\circledR}$ recording units were installed on the northern branch of the $9^{\text {th }}$ Polymylos bridge (on deck level at the middle of the total bridge deck), each supporting 12 uniaxial Kinemetrics Episensor (® accelerometers $( \pm 2 \mathrm{~g}$ full scale) installed on both sides of the bridge deck. The 
recording units have a 19-bit resolution, a sampling rate capacity of up to 200sps and a dynamic range of $108 \mathrm{~dB} @ 200$ sps. Fifteen sensors were installed on the deck, three on the basement of the central pier and three on each of the two abutments (at the support level of the elastomeric bearings), as shown in Figure 2a. Thus, the nine sensors monitor the earthquake-induced excitations at the two abutments and the basement of the pier. The particular layout of the instrumentation permits the analysis of both ambient and earthquake-induced response of the bridge. The 3 to 4-letter sensor labels follow the following convention: The last letter denotes the orientation of the uniaxial sensor (L: longitudinal, T: transverse, V: vertical). The previous one denotes the side of the bridge deck on which the sensor lies (R: right, L : left). Finally, the first one or two letters denote the bridge section that the sensor lies on (first letters U1 and U3 refer here to the abutment level where the elastomeric bearings are seated, U2 refers to the base of the central pier and all other letters refer to positions on the level of the bridge deck). The numbers next to each sensor label denotes the length of the cable used to connect the sensor to each recording unit. Among the 15 accelerometers located on the bridge deck, 8 record in the vertical, 1 in the longitudinal and the rest 6 in the transverse direction.

A similar, 24-accelerometer special array was used for the instrumentation of the southern branch of the 2nd Kavala Bypass Ravine Bridge. The two recording units were installed one at each deck side, at the middle of the total bridge span. The accelerometers were installed on both edges of the bridge deck (external sidewalk and internal New Jersey barrier). As shown in Figure 2b, of the 24 sensors, 18 were installed on the deck and 2 at the top of each of the three piers ( 6 in all) next to the elastomeric bearings so that adequate information is provided to distinguish between the pier and bearing stiffness. The sensor labels in Figure $2 b$ follow the same convention used for the previous instrumentation case (Figure 2a): letters $\mathrm{U} 1, \mathrm{U} 2$ and $\mathrm{U} 3$ refer to the top of the piers, all the rest refer to positions on the deck of the bridge. The particular layout of the instrumentation permits the recording of the dynamic response of the bridge under ambient (traffic and wind) loads.

Both recording systems have common start / common trigger capabilities to enable synchronous data acquisition. The trigger threshold can be set independently for each sensor, and the user can define the sensors that will cause 
a system trigger. The systems are equipped with GPS boards as well as with external GSM/GPRS cellular modems that allowed telematic control and data transfer to the user offices.

\section{Identification of structural modes}

\subsection{Ambient vibrations}

The estimation of the modal characteristics using ambient vibration data is based on a least squares minimization of the measure of fit

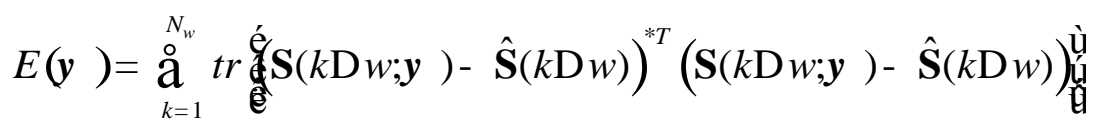

between the cross power spectral density (CPSD) matrix $\hat{\mathbf{S}}(k \mathrm{D} w) \hat{\mathrm{I}} C^{N_{0}{ }^{\prime} N_{0}}$ estimated from the measured output acceleration time histories and the CPSD matrix $\mathbf{S}(k \mathrm{D} w ; \boldsymbol{y}) \hat{I} C^{N_{0}{ }^{\prime} N_{0}}$ predicted by a modal model, where $N_{0}$ is the number of measured degrees of freedom (DOF), $\mathrm{D} w$ is the discretization step in the frequency domain, $k=\left\{1, \cdots, N_{w}\right\}$ is the index set corresponding to frequency values $w=k \mathrm{D} w, N_{w}$ is the number of data in the indexed set, and $\boldsymbol{y}$ is the parameter set to be estimated. Assuming general non-classically damped modes, the CPSD matrix $\mathbf{S}(k \mathrm{D} w ; \boldsymbol{y})$ based on the modal model of the structure is given by (Gauberghe 2004)

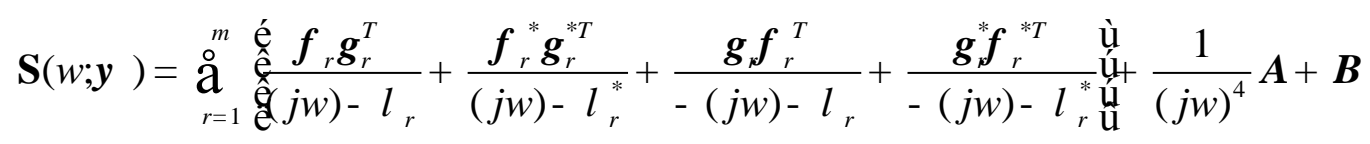

where $m$ is the number of contributing modes in the frequency range of interest, $l_{r}=-z_{r} w_{r} \pm j w_{r} \sqrt{1-z_{r}^{2}}$ is the complex eigenvalue of the $r$-th contributing mode, $w_{r}$ is the $r$-th modal frequency, $z_{r}$ is the $r$-th modal damping ratio, $\boldsymbol{f}_{r} \hat{\mathrm{I}} C^{N_{0}}$ is the complex modeshape of the $r$-th mode, $\boldsymbol{A} \hat{\mathrm{I}} R^{N_{0}^{\prime} N_{0}}, \boldsymbol{B} \hat{\mathrm{I}} R^{N_{0}^{\prime} N_{0}}$ are real symmetric matrices accounting for the contribution of the out-of-bound modes to the selected frequency range of interest, and $\boldsymbol{g}_{r} \hat{\mathrm{I}} C^{N_{0}}$ are vector quantities that depend on the characteristics of the modal model and the CPSD of 
the white noise input vector, while the symbol $u^{*}$ denotes the complex conjugate of a complex number $u$.

The modal parameter set $\boldsymbol{y}$ to be identified contains the parameters $w_{r}, z_{r}$, $\boldsymbol{f}_{r}, \boldsymbol{g}_{r}, r=1, \cdots, m, \boldsymbol{A}$ and $\boldsymbol{B}$ that completely define the CPSD matrix in eq. (2). The total number of parameters is $2 m\left(1+2 N_{0}\right)+N_{0}^{2}+N_{0}$ for non-classically damped modal models.

The minimization of the objective function (1) can be carried out efficiently, significantly reducing computational cost, by recognizing that the error function in (1) is quadratic with respect to the complex modeshapes $\boldsymbol{f}_{r}$ and the elements in the matrices $\boldsymbol{A}$ and $\boldsymbol{B}$. This observation is used to develop explicit expressions that relate the parameters $\boldsymbol{f}_{r}, \boldsymbol{A}$ and $\boldsymbol{B}$ to the vectors $\boldsymbol{g}_{r}$, the modal frequencies $w_{r}$ and the damping ratios $z_{r}$, so that the number of parameters involved in the optimization is reduced from $2 m\left(1+2 N_{0}\right)+N_{0}^{2}+N_{0}$ to $2 m\left(N_{0}+1\right)$. This reduction is considerable for a relatively large number of measurement points. Applying the optimality conditions in eq. (1) with respect to the components of $\boldsymbol{f}_{r}, \boldsymbol{A}$ and $\boldsymbol{B}$, a linear system of equations results for obtaining $\boldsymbol{f}_{r}, \boldsymbol{A}$ and $\boldsymbol{B}$ with respect to the $\boldsymbol{g}_{r}, w_{r}$ and $z_{r}, \quad r=1, \cdots, m$. The resulting nonlinear optimization problem with respect to the remaining variables $\boldsymbol{g}_{r}, w_{r}$ and $z_{r}$, $r=1, \cdots, m$, is solved in Matlab using available gradient-based optimisation algorithms.

The starting values required in the optimization are obtained from a two-step approach as follows. In the first step, conventional least squares complex frequency algorithms (Verboten 2002) are employed, along with stabilization diagrams, to obtain estimates of the modal frequencies $w_{r}$ and modal damping ratios $z_{r}$ and distinguish between the physical and the mathematical modes. These values in most cases are very close to the optimal values. In the second step, given the values of $w_{r}$ and $z_{r}$, the values of the residue matrices $\boldsymbol{R}_{r}=\boldsymbol{f}_{r} \boldsymbol{g}_{r}^{T} \hat{\mathrm{I}} C^{N_{0}{ }^{\prime} N_{0}}$ in eq. (2) are obtained by first recognizing that the objective function in (1) is quadratic with respect to $\boldsymbol{R}_{r}, \boldsymbol{A}$ and $\boldsymbol{B}$, then formulating and solving the resulting linear system of equations for $\boldsymbol{R}_{r}, \boldsymbol{A}$ and $\boldsymbol{B}$, and finally applying 
singular value decomposition to obtain estimates of $\boldsymbol{f}_{r}$ and $\boldsymbol{g}_{r}$ from $\boldsymbol{R}_{r}$. Usually, this two-step approach gives results that are very close to the optimal estimates. However, for closely-spaced and overlapping modes it is often recommended to solve the original nonlinear optimization problem with respect to $\boldsymbol{g}_{r}, w_{r}$ and $z_{r}$, $r=1, \cdots, m$, using the estimates of the two-step approach as starting values.

\subsection{Earthquake-induced vibrations}

The methods developed by McVerry (1980) in the frequency domain and Beck and Jennings (1980) in the time domain have been extended in this work to treat non-classically damped modal models, since damping may not be proportionally distributed in various structural components, especially due to the energy dissipation mechanism provided locally by the elastomeric bearings and the foundation soil. The modal identification methodology also accounts for different support excitations encountered in bridges. The modal identification carried out in the frequency domain using earthquake vibration data is next outlined. The modal identification method is based on a minimization of the measure of fit between the Fourier transform $\hat{\ddot{y}}(k \mathrm{D} w) \hat{\mathrm{I}} C^{N_{0}}$ of the measured acceleration response vector and the Fourier transform $\ddot{\boldsymbol{y}}(k \mathrm{D} w ; \boldsymbol{y}) \hat{\mathrm{I}} C^{N_{0}}$ of the acceleration responses predicted from a parameterized modal model of the structure. The Fourier transform vector $\ddot{\boldsymbol{y}}(k \mathrm{D} w ; \boldsymbol{y})$ of the acceleration responses based on the nonclassically damped modal model can be obtained in the form

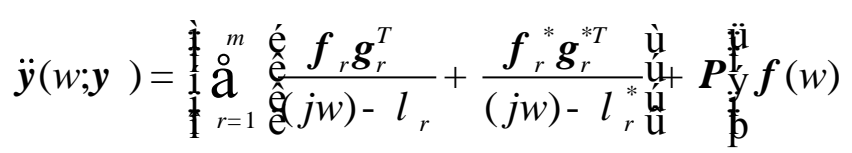

where the vector $\boldsymbol{f}(w)$ contains the Fourier transforms of the input acceleration time histories.

The parameter set $\boldsymbol{y}$ to be identified contains the modal frequencies $w_{r}$, the modal damping ratios $z_{r}$, the complex modeshapes $\boldsymbol{f}_{r}$, the modal participation factor vectors $\boldsymbol{g}_{r}, \quad r=1, \cdots, m$, and the constant matrix $\boldsymbol{P}$ which contains the pseudostatic response of the structure due to multiple differential base excitations. The total number of parameters is $2 m\left(1+N_{0}+N_{\text {in }}\right)+N_{0} N_{\text {in }}$ for non-classically damped modal models. The formulation in eq. (3) assumes that measured 
vibrations start from zero. Non-zero starting vibration levels have also been accounted for by adding additional terms in eq. (3) and augmenting the parameter set $\boldsymbol{y}$ to include parameters that account for the initial conditions of the modal equations. It should be noted that a similar modal identification method has been developed in the time domain based on minimizing a measure of fit between the measured acceleration response vector and the acceleration response vector predicted from a parameterized non-classically damped modal model.

The optimal values of the parameter set $\boldsymbol{y}$ are obtained by minimizing an objective function similar to (1) with the CPSDs replaced by the Fourier transform vectors. Similar to the CPSD case, the number of the design variables can be reduced to $w_{r}, z_{r}$ and $\boldsymbol{g}_{r}$ by recognizing that the objective function in (1) is quadratic with respect to the complex modeshapes $\boldsymbol{f}_{r}$ and the real matrix $\boldsymbol{P}$. Applying the optimality conditions in eq. (1) with respect to the components of $f_{r}$ and $\boldsymbol{P}$, a linear system of equations results for obtaining $\boldsymbol{f}_{r}$ and $\boldsymbol{P}$ with respect to the vectors $\boldsymbol{g}_{r}, w_{r}$ and $z_{r}$. The resulting nonlinear optimization problem with respect to the $2 m\left(3+N_{i n}\right)$ parameters $\boldsymbol{g}_{r}, \quad w_{r}$ and $z_{r}, r=1, \cdots, m$, is solved in Matlab using available gradient-based optimization algorithms. The derivatives of the objective function with respect to the modal parameters are evaluated analytically, accelerating the convergence of the algorithm. Modal sweep approaches (Werner et al. 1987) are also implemented to improve the effectiveness of the proposed algorithm.

\section{Finite element model updating methodology}

The objective in a finite element model updating methodology is to estimate the values of the structural parameter set $\boldsymbol{q} \hat{\mathrm{I}} R^{N_{q}}$ of a class of linear finite element models so that the modal frequencies and modeshapes $\left\{w_{r}(\boldsymbol{q}), \boldsymbol{f}_{r}(\boldsymbol{q}) \hat{\mathrm{I}} R^{N_{0}}, r=1, \cdots, m\right\}$ predicted by the linear class of models best matches, in some sense, the experimentally obtained modal data $\left\{\hat{w}_{r}, \hat{\boldsymbol{f}_{r}} \hat{\mathrm{I}} R^{N_{0}}, r=1, \cdots, m\right\}$ contained in the set $\boldsymbol{y}$, where $m$ is the number of observed modes, and $N_{0}$ is the number of recorded DOFs. The optimal values of the parameter set $\boldsymbol{q}$ are obtained by minimizing the weighted modal residuals (Christodoulou and Papadimitriou 2007) 


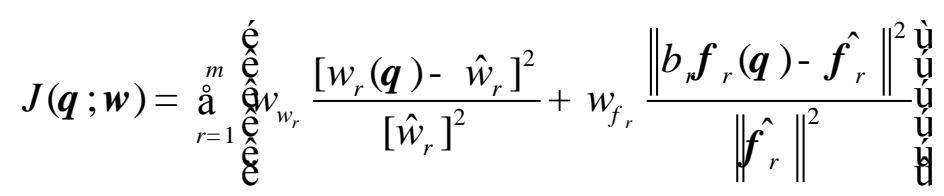

The first norm in eq. (4) represents the difference between the measured and the model predicted frequency for the $r$-th mode, while the second norm represents the difference between the measured and the model predicted modeshape components for the $r$-th mode, where $b_{r}={\hat{\boldsymbol{f}_{r}^{T}}}_{r}^{T}(\boldsymbol{q}) / \boldsymbol{f}_{r}^{T}(\boldsymbol{q}) \boldsymbol{f}_{r}(\boldsymbol{q})$ is a normalizing scalar guaranteeing that the measured $\hat{\boldsymbol{f}_{r}}$ is closest to $\boldsymbol{f}_{r}(\boldsymbol{q})$ for given $\boldsymbol{q}$. The weighting factors $w_{w_{r}}{ }^{3} 0$ and $w_{f_{r}}{ }^{3} 0, r=1, \cdots, m$, satisfy the condition $\stackrel{\mathrm{a}_{r=1}^{m}}{{ }^{m}}\left[w_{w_{r}}+w_{f_{r}}\right]=1$. The objective function $J(\boldsymbol{q} ; \boldsymbol{w})$ represents an overall measure of fit between the measured and the model predicted modal characteristics. The sensitivity of the updated models to the choice of the weight values has been studied in Christodoulou and Papadimitriou (2007). Herein, conventional weighted least squares methods are used which assume equal weight values.

The optimization of $J(\boldsymbol{q} ; \boldsymbol{w})$ in eq. (4) with respect to $\boldsymbol{q}$ can readily be carried out numerically using any available gradient-based algorithms for optimizing a nonlinear function of several variables. In the case of multiple local/global optima, a hybrid optimization algorithm (Christodoulou and Papadimitriou 2007) is used that exploits the advantages of random search evolutionary strategies (Beyer 2001) and gradient-based methods. Specifically, an evolution strategy is used to explore the parameter space and detect the neighborhood of the global optimum, avoiding premature convergence to a local optimum. Then the method switches to a gradient-based algorithm starting with the best estimate obtained from the evolution strategy and using gradient information to accelerate convergence to the global optimum.

\section{Structural modal identification of Polymylos and Kavala bridges}

Modal identification results (modal frequencies and modal damping ratios) for the Polymylos and Kavala bridges are shown in Table 1 for ambient vibration (AV) data of approximately 30 minutes duration each. Also, for the Polymylos bridge, 
modal identification results are shown in Table 1 for a low level, magnitude $M_{L}=4.6$, earthquake event that occurred on 21/2/2007 (2:04:38 GMT) at a distance $35 \mathrm{~km}$ Northeast of the bridge. The $20 \mathrm{sec}$ earthquake recordings are clear and not hidden by the ambient vibrations since the latter are much smaller in amplitude due to the fact that the earthquake occurred after midnight, so the traffic of the bridge was scarce. It is noted that eight (8) modes were successfully and reliably identified for the Polymylos bridge: five transverse modes and three bending modes. From the earthquake vibration (EV) data, in addition to these modes, one more longitudinal mode was identified. Representative measured modeshapes $\left(1^{\text {st }}\right.$ bending and $2^{\text {nd }}$ transverse) are shown in Figure 3 for the Polymylos bridge. For the Kavala bridge, seven modes were reliably estimated from the AV data: two transverse, one longitudinal, and four closely-spaced bending modes. The four closely-spaced modes, predicted also by the design finite element model of the structure, are due to the weak connection of the four spans through the 2-meter-long 20cm-thick continuity slabs described in Section 2.

Comparing the modal damping ratios in Table 1, it is observed that the bending modes have significantly lower values of damping, of the order of $0.4 \%$ to $0.7 \%$ for the Polymylos and Kavala bridges, than the damping values of the lower transverse and longitudinal modes which are of the order of $0.8 \%$ to $5.9 \%$ for the Polymylos bridge and of the order of $1.4 \%$ to $5.6 \%$ for the Kavala bridge. The higher damping values observed for the lower transverse and longitudinal modes can be attributed to the energy dissipation arising from the higher modal deformation levels of the elastomeric bearings at the ends of the bridges which dominate the motion of these modes. Also, soil damping could also have contributed to the higher damping values observed for these modes.

For the Polymylos bridge, Table 2 compares the measured peak acceleration responses and the root mean square (RMS) acceleration responses for the ambient and the earthquake excitations. The results for only twelve out of the fifteen sensors installed on the deck are reported in Table 2 since three out of the eight sensors recording along the vertical direction malfunctioned during the earthquake event. Also, Table 2 reports the ratio between the ambient and the earthquake vibrations for the peak and the RMS responses for each output channel. Figure 4 gives a comparison between the ambient and the earthquake acceleration time 
histories for two representative recordings along the vertical (sensor B2RV) and the transverse (sensor SRT) directions.

From the Polymylos results in Table 1, it is observed that the modal frequencies due to earthquake vibrations are $4 \%$ to $15 \%$ higher than the modal frequencies identified from the ambient vibrations. No conclusive explanation can be given for these differences without making assumptions about the bridge behavior within the measured vibration levels given in Table 2 and Figure 4. These differences could be attributed to the nonlinear softening hysteretic behavior of the structural components, especially the elastomeric bearings. The results in Table 2 reveal that the peak acceleration responses for the earthquake induced vibrations are 1.4 to 3.8 times lower than the peak acceleration responses of the ambient vibrations. Accepting that the estimation of the equivalent modal frequencies is dominated by the peak vibration levels, this could justify a higher secant stiffness of the elastomeric bearings for the lower earthquake peak vibration levels which results in stiffer structures and thus justifies the increase in the equivalent values of the modal frequencies observed in Table 1 for earthquake induced vibrations. However, this explanation cannot be used to justify the higher modal frequency values observed for the modes associated with bending of the deck since these modes are not affected by the bearing stiffness. It is unlikely that similar softening nonlinear effects will arise by the deformation of the pier and deck elements in these low vibration levels.

To further support the above argument, the values of the modal frequencies were also identified using much shorter duration segments of the ambient vibrations recordings shown in Figure 4, selected so that the peak acceleration levels are approximately the same as or smaller than the peak acceleration of the earthquake recordings. The estimated values of the modal frequencies obtained by analyzing these short duration segments were found to be almost identical to the values of the modal frequencies that were estimated using the whole, approximate 30 minutes in duration, segment of the records shown in Figure 4. This verifies that at the low vibration levels considered, the aforementioned differences in the peak acceleration levels between the ambient and the earthquake induced vibrations cannot justify the large differences in the modal frequencies observed in Table 1. 
In contrast to the peak vibration levels, the levels of the RMS response in Table 2 of the approximately 30 minutes ambient acceleration measurements are 0.25 to 0.82 times the corresponding root mean square earthquake response levels. Accepting that the estimation of the equivalent modal frequencies in Table 1 is dominated by the RMS vibration levels, the modal frequencies due to higher RMS earthquake vibration levels are expected to decrease if softening of the elastomeric bearings takes place, which is not consistent with the opposite increasing trend observed in Table 1.

A more reasonable explanation that can account for the differences in the identified values of the modal frequencies in Table 1 is soil structure interaction effects (Safak 1995). Specifically, in the earthquake vibration case, the modal properties of the system were identified using as input accelerations the nine recordings at the two abutments and the base of the central pier and as output accelerations the twelve available recordings along the bridge deck. Thus, ignoring the rigid body rotation of the central pier foundation at the low vibration levels measured, the modal frequencies identified by the input-output earthquake vibration measurements are those of the fixed-base bridge. The effects of soilstructure interaction have been "filtered out" since the base motion of the abutment and the pier foundation were used as input accelerations in the modal identification process. In contrast, for the ambient vibration case, the modal properties of the system, obtained from the ambient measurements due to excitations from the traffic and wind loads, were identified using only the twelve output accelerations recorded along the bridge deck. Thus, the modal frequencies due to ambient vibrations correspond to the dynamic characteristics of the combined system consisting of the bridge and accounting for soil-structure interaction effects. This interaction effect is due to the additional soil flexibility provided at the base supports of the bridge. The presence of this effect is also supported from the non-zero vibration levels recorded at the base of the pier and the top of the side abutments during ambient measurements. Thus, soil-structure interaction effects cause the combined soil-foundation-superstructure system to appear as less stiff than the superstructure (fixed-base bridge) itself, resulting in lower values of the modal frequencies which is consistent with the results observed in Table 1. 
Figure 5 compares the measured power spectral densities with the power spectral densities predicted by the identified optimal modal models for selected sensors for the Polymylos bridge (Figure 5a) and the Kavala bridge (Figure 5b) zoomed at the frequency range near the four closely-spaced and overlapping modes. As it is seen, the fit of the measured power spectral density is very good which validates the effectiveness of the proposed modal identification software based on ambient vibrations. Figure 6 compares the Fourier transform (FT) of the earthquake-induced accelerations and the FT of the accelerations predicted by the optimal modal model for selected sensors. A very good fit is also observed, validating the effectiveness of the proposed modal identification software based on earthquake recordings.

\section{Finite element model updating of Polymylos and Kavala bridges}

Detailed finite element models were created that correspond to the model used for the design of the two bridges. The models were constructed based on the material properties and the geometric details of the structures. The entire simulation is performed within the COMSOL Multi-physics (COMSOL 2005) modeling environment. The finite element (FE) model for the Polymylos bridge is created using three-dimensional two-node beam-type finite elements to model the deck and the piers, and spring elements to model the bearings. This model is shown in Figure $7 \mathrm{a}$ and has 1350 degrees of freedom. The FE model of the Kavala bridge is simulated using three-dimensional two-node beam-type finite elements exclusively to model the deck, the piers and the bearings. This model is shown in Figure $7 \mathrm{~b}$ and has 900 degrees of freedom. Each span consists of four longitudinal beams, representing the post-tensioned beams supporting the deck, and six transverse beams, which form a horizontal grid. The cross-sectional parameters of each one of the longitudinal beam elements are those of an "equivalent" crosssection that accounts for the section of the post-tensioned beam, as well as the corresponding effective width of the deck plate. The transverse beams at the two ends of the span correspond to the existing cross-beams above the bearings, whereas the other four transverse beams represent the coupling of the longitudinal beams in the transverse direction due to the presence of the deck. Adjacent spans are interconnected with a 20-cm-thick 2-meter-long plate, which is also simulated 
in the finite element model. In both bridge models, the abutments are considered as non-deformable (rigid foundation), whereas the piers are assumed to be rigidly connected to the foundation, ignoring soil structure interaction effects to the dynamics of the bridge systems.

For comparison purposes, Table 1 lists the values of the modal frequencies predicted by the nominal design finite element models (FEM). Comparing with the identified modal frequency values it can be seen that the design FEM-based modal frequencies are significantly lower than the identified modal frequencies. This is partly due to the fact that the design finite element model was constructed taking into account the high vibration levels associated with the strong design earthquake prescribed by the seismic code, while the ambient vibrations are very low amplitude vibrations. During low amplitude vibrations, the structure is found to be much stiffer as predicted by the identified modal frequencies. In order to identify the main sources of stiffness increase in the case of low amplitude ambient and earthquake-induced vibrations, the finite element models are next updated using the identified modal properties in Table 1.

The parameters in the set $\boldsymbol{q}$ to be updated are stiffness related parameters that scale the contribution of the nominal values of stiffness properties in the initial finite element model. Thus, the nominal finite element model corresponds to parameter values $\boldsymbol{q}=\mathbf{1}$. For the Polymylos bridge, one parameter scales the stiffness $E$ of the elastomeric bearings along the transverse directions, two parameters scale independently the modulus of elasticity $E$ of the deck and pier beam elements that affect the deck and pier stiffness, respectively, and four parameters scale independently the cross-sectional moment of inertias $I_{x x}, I_{y y}$ and $I_{z z}$ of the deck and top pier beam elements with respect to the global coordinate axes shown in Figure 7a. For the Kavala bridge, one parameter scales the modulus of elasticity of the deck that affects the deck stiffness, one parameter scales the stiffness of the three piers assumed to be fully correlated, and one to two parameters scale the stiffness of the bearings assumed to be either fully correlated or the stiffness of the pier bearings to vary independently of the stiffness of the abutment bearings.

Model updating results for the Polymylos and the Kavala bridges are presented in Table 3 using the available ambient and earthquake-induced modal data in Table 1. For the Polymylos bridge, the modal properties fitted are the lowest three 
transverse modes and the lowest two bending modes. For the Kavala bridge, the modal properties fitted are the lowest two transverse modes, the lowest longitudinal mode and the lowest four closely-spaced bending modes. The model updating results are presented for a different combination of parameters. Case 1 and Case 2 differ by the number of parameters used for model updating. Each case is distinguished by subcases A and B. In subcase A both modal frequencies and modeshape components of the involved modes are updated simultaneously, while in subcase B only the modal frequencies of the involved modes are updated.

Results for the percentage error $\Delta \omega$ between the values of the measured modal frequencies and the modal frequencies predicted by the optimal finite element model for the Polymylos bridge are shown in Table 4 for the cases considered in Table 3. Similar results for the MAC (Modal Assurance Criterion) values between the measured modeshapes and the modeshapes predicted by the optimal finite element model are also shown. The closer the value of the MAC is to one, the closer the fit between the measured and model predicted modeshapes. From the percentage error values in the modal frequencies presented in Table 4 , it can be observed that a very good or even an exact fit in the modal frequencies is obtained when only the modal frequencies are included in the updating process (Cases B). Including also the modeshape components in the updating process, a trade-off in the fit between the modal frequencies and modeshapes is observed by comparing the $\Delta \omega$ and MAC values between Cases A and B. Specifically, the fit in the modeshape components is improved as it is evident by the overall increase in the MAC values to values closer to one, at the expense of deteriorating the fit in the modal frequencies observed by the increase in the percentage error values $\Delta \omega$.

It is worth noting that for all cases considered for the Polymylos bridge, the stiffness values of the elastomeric bearings for the ambient vibrations are approximately 3.2 to 3.5 times the nominal design values and for the earthquake vibrations are approximately 3.7 to 3.9 times the nominal design values. The identified values of the bearing stiffness are much higher than the nominal design values due to the highly nonlinear hysteretic force-displacement relationship of the elastomeric bearings. At low strain levels experienced by the bearings due to low amplitude ambient and earthquake-induced excitations, their secant stiffness is much higher than the one at the significantly much higher strain levels associated with the design of the bearings for the strong design earthquakes 
prescribed by the seismic code. These large differences of the elastomeric bearings stiffnesses between the identified and the design ones are also consistent with the results obtained by Chaudhary et al. (2002) for base-isolated bridges and by Stewart et al. (1999) for base-isolated buildings. Qualitatively similar results are observed for the bearing stiffness values in Table 3 for the Kavala bridge, although these values are significantly higher than those identified for the Polymylos bridge.

Another issue worth pointing out is the very good fit of the modal frequencies in the range of $0.4 \%$ to $2.0 \%$ obtained from the updated finite element model for the case of five parameters (Case 2-A) and earthquake measurements as compared to a much worse fit, as high as $4.5 \%$ in at least three modes, for the Case 2-A of ambient vibrations. The good fit for the earthquake case validates the finite element model used, while the large errors for the ambient vibration case suggest that the model is inadequate, probably due to soil structure interaction effects ignored in the finite element modeling of the bridge. This observation supports once more the contributing effect of the soil structure interaction in the dynamics of the identified system for the ambient vibration case.

For the case of ambient vibrations, the bending stiffness values of the deck vary from 0.90 to 1.12 its nominal design values, depending on the bending axes, while the stiffness values of the piers are approximately closer to the nominal design values of 1.00 for the cases 1 -A and 2-A. For the case of earthquake vibrations, the bending stiffness values with respect to the global $y$ axis (Figure 7) along the transverse direction of the bridge are very close or slightly higher (up to $4 \%$ ) than their nominal values. The bending stiffness values with respect to the global $z$ axis for the deck beam elements and global $x$ axis for the pier beam elements are approximately 1.5 times their nominal values. These latter bending stiffness along with the stiffness of the bearings provide the main resistant mechanism to the transverse motion of the bridge. The $50 \%$ increase in the identified stiffness values from the nominal design values is within the levels expected for the piers and the columns and are attributed to the low strain levels experienced by the deck and the piers due to motion in the transverse direction. 


\section{Conclusions}

The proposed modal identification methods for ambient and earthquake-induced low level vibrations reliably identified the lower transverse, longitudinal and vertical modes of the two representative bridges of the Egnatia Odos motorway in Greece, included four-closely spaced and overlapping bending modes for the Kavala bridge. The damping values of the bending modes are of the order of $0.4 \%$ to $0.7 \%$ which is significantly lower than the damping values of the lower transverse and longitudinal modes. This is attributed to the higher damping provided mostly by the elastomeric bearings and the soil for the latter modes. The modal frequencies due to earthquake-induced vibrations are found to be $4 \%$ to $15 \%$ higher than the modal frequencies due to ambient vibrations. This is attributed mainly to the soil-structure interaction effects contributing to the dynamics of the bridge systems during excitation from wind and traffic loads. These effects are not present in the identified dynamics of the system based on the earthquake induced-vibrations due to the use of the input acceleration measurements at the base of the piers and the abutments. Finally, the identified values of the modal frequencies of the two structures are significantly higher than the modal frequencies suggested by the design finite element model. The updated finite element models based on the identified modal properties present clear evidence that these differences are mostly due to the much higher stiffness values of the elastomeric bearings at the low amplitude vibration levels actually experienced by the bridges. This dynamic behaviour is consistent for both bridges and is attributed to the highly nonlinear softening hysteretic behaviour of the laminated elastomeric bearings. Specifically, the secant stiffness of the bearings for low deformation (strain) levels under the low magnitude vibrations associated with the measurements is significantly higher than the one that corresponds to the high deformation levels associated with the strong design earthquake prescribed by the seismic code.

Acknowledgements: This research was funded by the General Secretariat of Research and Technology of Greece and the European Union (European Social Fund), through the EPAN program ASProGe (Seismic Protection of Bridges) under grant DP15. 


\section{References}

Basseville M, Benveniste A, Goursat M, Hermans L, Mevel L, Van der Auweraer H (2001) Output-only subspace-based structural identication: from theory to industrial testing practice. ASME Journal of Dynamic Systems Measurement and Control 123(4): 668-676.

Beck JL (1978) Determining models of structures from earthquake records. Report No. EELRL 78-01, Earthquake Engineering Research Laboratory, California Institute of Technology, Pasadena, CA.

Beck JL, May BS, Polidori DC (1994) Determination of modal parameters from ambient vibration data for structural health monitoring. Proceedings of the 1st World Conference on Structural Control, Los Angeles, USA pp. 1395-1402.

Beck JL, Jennings PC (1980) Structural identification using linear models and earthquake records. Earthquake Engineering and Structural Dynamics 8: 145-160.

Beyer HG (2001) The theory of evolution strategies. Berlin, Springer-Verlag.

Bohle K, Fritzen CP (2003) Results obtained by minimizing natural frequencies and MAC-value errors of a plate model. Mechanical Systems and Signal Processing 17: 55-64.

Brincker R, Zhang L, Andersen P (2001) Modal identification of output-only systems using frequency domain decomposition. Smart Materials and Structures 10: 441-445.

Christodoulou K, Papadimitriou C (2007) Structural identification based on optimally weighted modal residuals. Mechanical Systems and Signal Processing 21: 4-23.

Chaudhary MTA, Abe M, Fujino Y (2000) System identification of two base-isolated buildings using seismic records. Journal of Structural Engineering (ASCE) 126(10): 1187-1195.

Chaudhary MTA, Abe M, Fujino Y (2002) Role of structural details in altering the expected seismic response of base-isolated bridges. Mechanical Systems and Signal Processing 16(2-3): 413-428.

COMSOL AB (2005) COMSOL Multiphysics User's Guide. [http://www.comsol.com/].

Gauberghe B (2004) Applied frequency-domain system identification in the field of experimental and operational modal analysis. PhD Thesis, Vrije Universiteit Brussel, Belgium.

Guillaume P, Hermans L, Van der Auweraer H (1999) Maximum likelihood identification of modal parameters from operational data. Proceedings of the International Modal Analysis Conference - IMAC 2, 1887-1893.

Katafygiotis LS, and Yuen K-V (2001) Bayesian spectral density approach for modal updating using ambient data. Earthquake Engineering and Structural Dynamics 30(8): 1103-1123.

Lam HF, Katafygiotis LS, Mickleborough NC (2004) Application of a statistical model updating approach on phase I of the IASC-ASCE structural health monitoring benchmark study. Journal of Engineering Mechanics (ASCE) 130 (1): 34-48.

McVerry GH (1980) Structural identification in the frequency domain from earthquake records. Earthquake Engineering and Structural Dynamics 8: 161-180.

Mottershead JE, Friswell MI (1993) Model updating in structural dynamics: A survey. Journal of Sound and Vibration 167: 347-375. 
Papageorgiou AS, Lin BC (1989) Influence of lateral-load-resisting system on the earthquake response of structures - A system identification study. Earthquake Engineering and Structural Dynamics 18: 799-814.

Peeters B, De Roeck G (1999) Reference-based stochastic subspace identification for output-only modal analysis. Mechanical Systems and Signal Processing 13(6): 855-878.

Peeters B, De Roeck G (2001) Stochastic system identification for operational modal analysis: A review. Journal of Dynamic Systems, Measurement and Control 123(4): 659-667.

Peeters B, Van der Auweraer H (2005) Recent developments in operational modal analysis. EURODYN 2005, C. Soize \& G.I Schueller (eds), Millpress, Rotterdam 149-154.

Safak E (1995) Detection and identification of soil-structure interaction in buildings from vibration recordings. Journal of Structural Engineering (ASCE) 121(5): 899-906.

Stewart JP, Conte JP, Aiken ID (1999) Observed behavior of seismically isolated buildings. Journal of Structural Engineering 125: 955-964.

Teughels A, De Roeck G, Suykens JAK (2003) Global optimization by coupled local minimizers and its application to FE model updating. Computers and Structures 81 (24-25): 2337-2351.

Verboven P (2002) Frequency domain system identification for modal analysis. PhD Thesis, Vrije Universiteit Brussel, Belgium.

Werner SD, Beck JL, Levine MB (1987) Seismic response evaluations of Meloland road overpass using 1979 Imperial Valley earthquake records. Earthquake Engineering and Structural Dynamics 15: 249-274. 


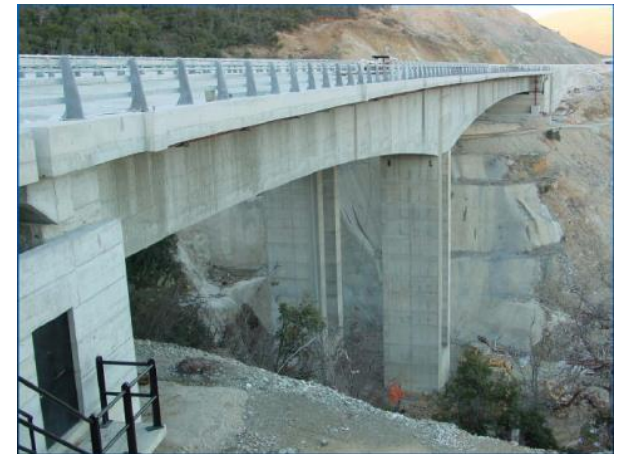

(a)

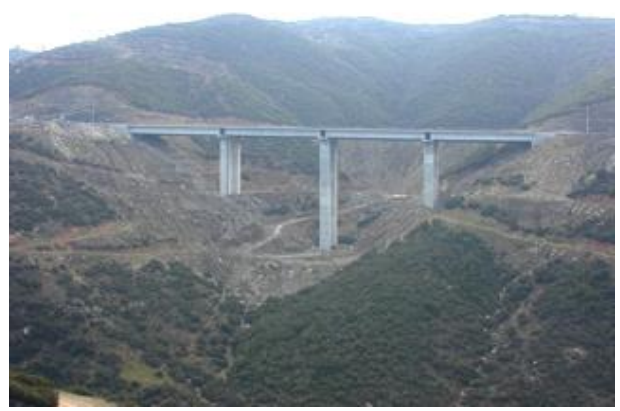

(b)

Figure 1: View of (a) $9^{\text {th }}$ Polymylos bridge, and (b) $2^{\text {nd }}$ Kavala bridge 


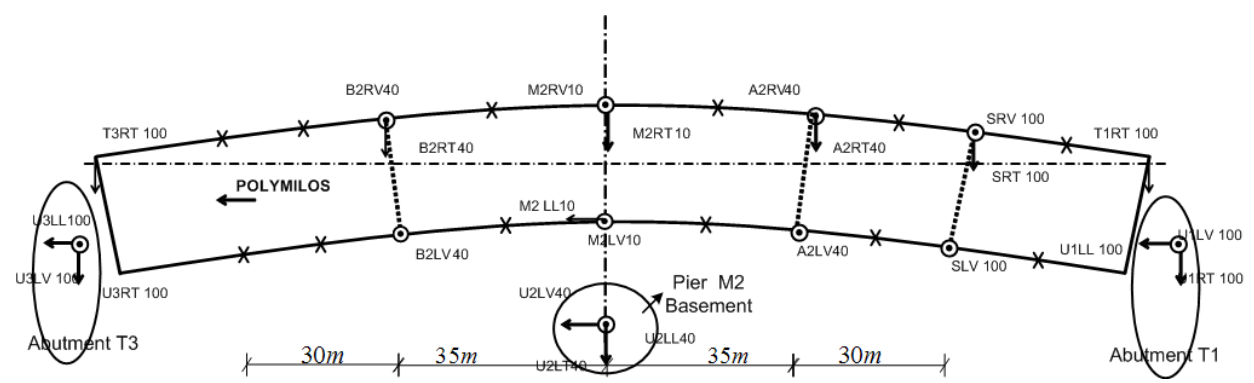

(a)

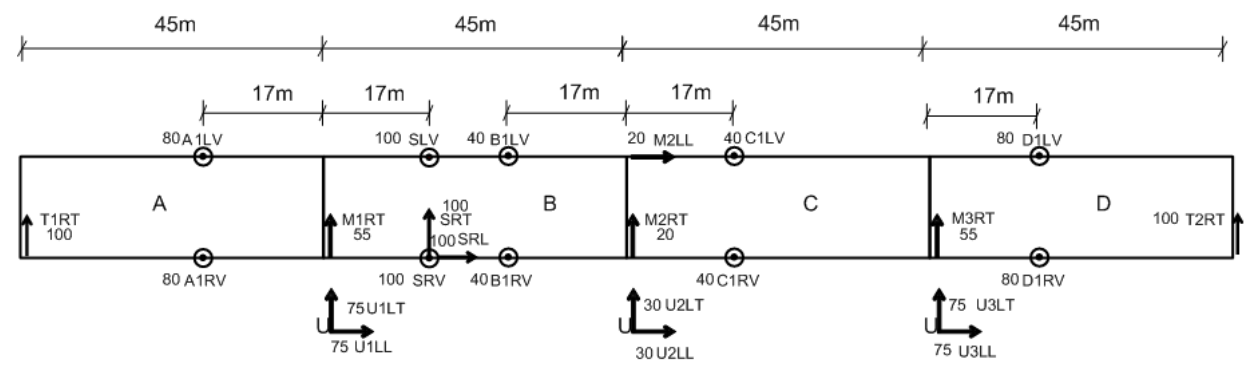

(b)

Figure 2: Instrumentation layout of (a) Polymylos and (b) Kavala bridges 


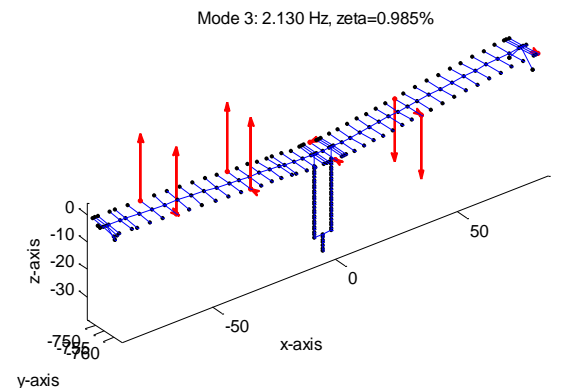

(a)

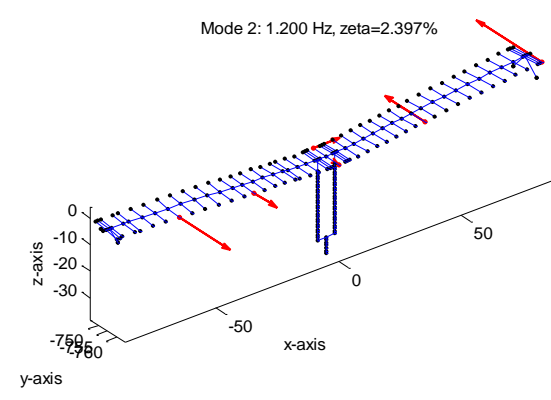

(b)

Figure 3: (a) $1^{\text {st }}$ bending and (b) $2^{\text {nd }}$ transverse modeshape of the Polymylos bridge 


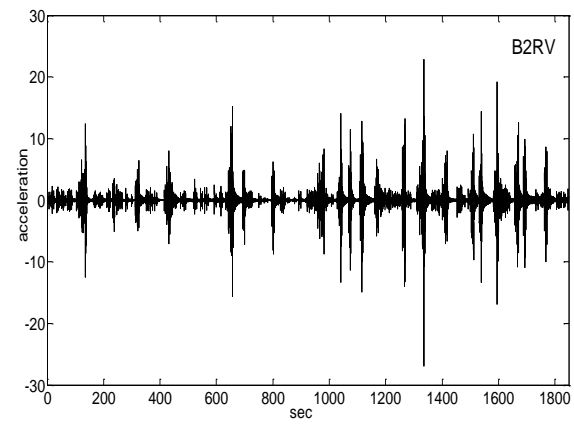

(a)

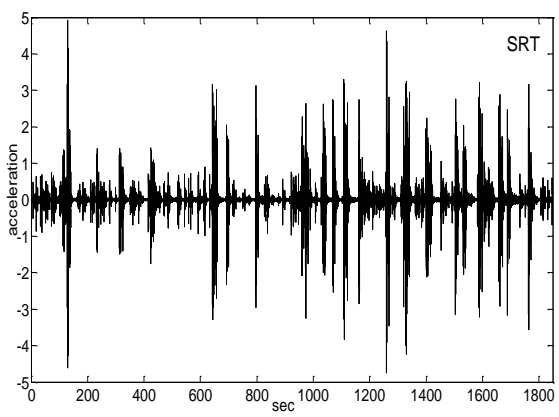

(c)

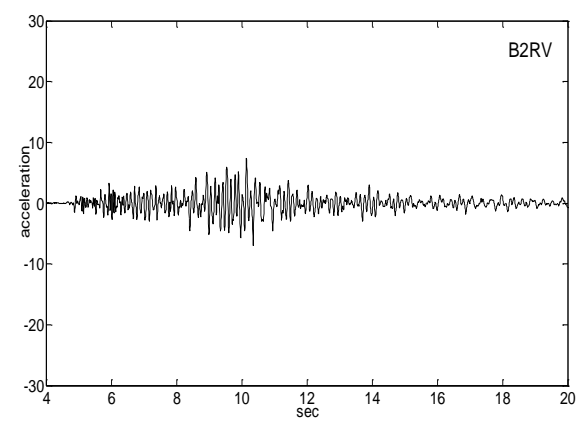

(b)

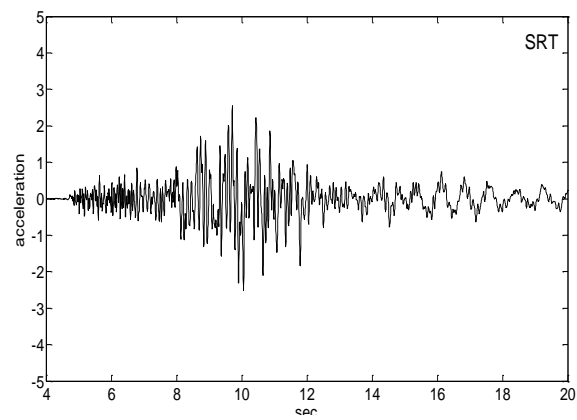

(d)

Figure 4: Accelerations time history measurements from ambient and earthquake vibrations at sensors B2RV and SRT, (a,c) ambient, (b,d) earthquake 


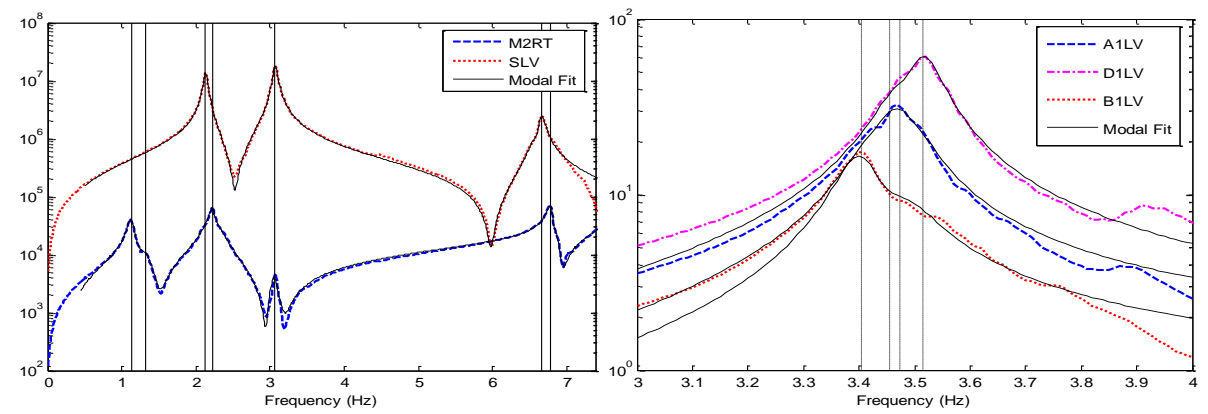

(a)

(b)

Figure 5: Comparison between measured and optimal modal model predicted power spectral densities for selected sensors (a) Polymylos bridge, and (b) Kavala bridge (zoomed at frequency range of four closely-spaced modes) 


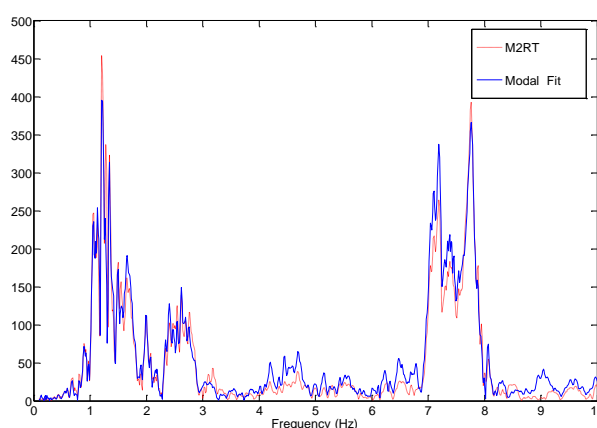

(a)

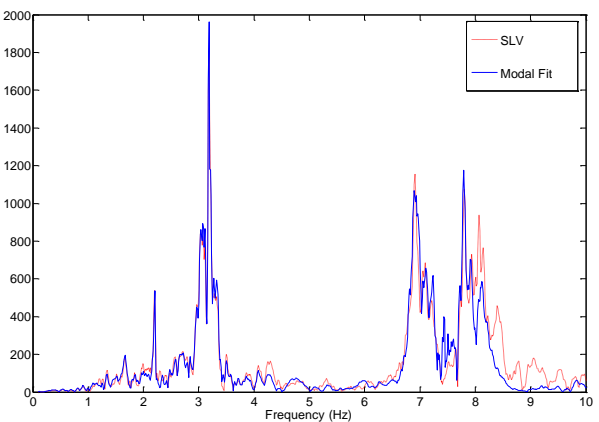

(b)

Figure 6: Comparison between measured and optimal modal model predicted Fourier transforms of the Polymylos earthquake accelerations recordings for selected sensors 


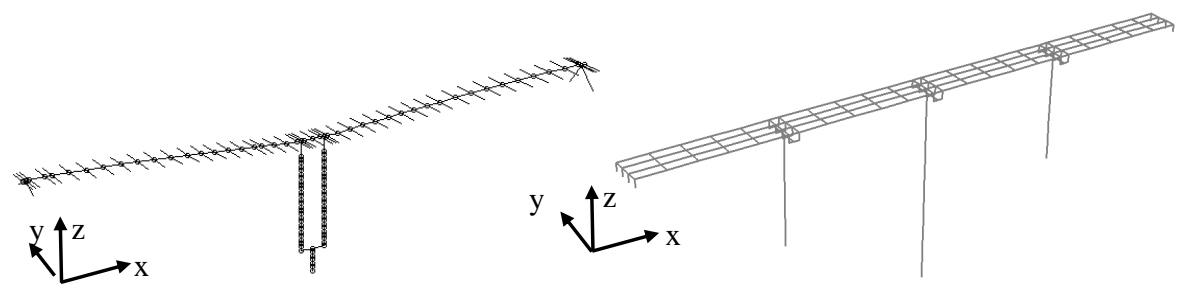

(a)

(b)

Figure 7: Finite Element Model of (a) Polymylos bridge, and (b) Kavala bridge 
Table 1: Identified and design FE model predicted modal frequencies $w$ and damping ratios $z$ $(\mathrm{AV} / \mathrm{EV}=$ Ambient/Earthquake Vibrations $)$

\begin{tabular}{|c|c|c|c|c|c|}
\hline \multirow{3}{*}{ Mode } & \multicolumn{3}{|c|}{ Polymylos Bridge } & \multicolumn{2}{|c|}{ Kavala Bridge } \\
\hline & AV & EV & FEM & AV & FEM \\
\hline & $w \quad \mathrm{~Hz} \quad z \quad(\%)$ & $w \mathrm{~Hz} \quad z \quad(\%)$ & $w \mathrm{~Hz}$ & $w \mathrm{~Hz} z \quad(\%)$ & $w \mathrm{~Hz}$ \\
\hline $1^{\text {st }}$ Transverse & $1.13 \quad 2.0$ & 1.29 & 0.96 & 0.81 & 0.53 \\
\hline $1^{\text {st }}$ Longitudinal & - & 1.17 & 0.70 & 1.29 & 0.57 \\
\hline $2^{\text {nd }}$ Transverse & 1.20 & 1.30 & 0.70 & 1.61 & 0.67 \\
\hline $1^{\text {st }}$ Bending (deck) & 2.13 & 2.20 & 2.18 & 3.40 & 2.78 \\
\hline $3^{\text {rd }}$ Transverse & 2.22 & 2.56 & 1.91 & - & - \\
\hline $2^{\text {nd }}$ Bending (deck) & 3.07 & 3.20 & 3.21 & 3.46 & 2.82 \\
\hline $4^{\text {th }}$ Transverse & 4.10 & 4.23 & 3.77 & - & - \\
\hline $3^{\text {rd }}$ Bending (deck) & 6.66 & 6.89 & 7.10 & 3.47 & 2.82 \\
\hline $5^{\text {th }}$ Transverse & 6.78 & 7.24 & 7.02 & - & - \\
\hline $4^{\text {th }}$ Bending (deck) & - & - & - & 3.51 & 2.83 \\
\hline
\end{tabular}


Table 2: Comparison of Peak and RMS acceleration response obtained from Ambient (AV) and Earthquake induced Vibrations (EV) for the Polymylos bridge

\begin{tabular}{|c|c|c|c|c|c|c|}
\hline & \multicolumn{2}{|c|}{ Peak response $\left(\mathrm{cm} / \mathrm{sec}^{2}\right)$} & \multicolumn{3}{c|}{ RMS $\left(\mathrm{cm} / \mathrm{sec}^{2}\right)$} \\
\hline Channel & AV & EV & AV/EV & AV & EV & AV/EV \\
\hline B2LV & 23.2470 & 7.1062 & 3.2714 & 0.9181 & 1.9397 & 0.4733 \\
\hline M2LL & 2.1767 & 1.0009 & 2.1747 & 0.0922 & 0.2407 & 0.3830 \\
\hline M2LV & 11.2310 & 2.9575 & 3.7975 & 0.6044 & 0.7350 & 0.8223 \\
\hline SLV & 15.9950 & 6.6148 & 2.4181 & 0.8847 & 2.0163 & 0.4388 \\
\hline T3RT & 5.9160 & 3.3652 & 1.7580 & 0.1825 & 0.7129 & 0.2561 \\
\hline B2RV & 26.9220 & 7.3206 & 3.6776 & 0.9704 & 1.7120 & 0.5668 \\
\hline B2RT & 7.7054 & 2.3919 & 3.2215 & 0.2928 & 0.6667 & 0.4392 \\
\hline M2RT & 4.3362 & 2.5179 & 1.7221 & 0.2582 & 0.6141 & 0.4204 \\
\hline A2RT & 5.5674 & 2.5210 & 2.2084 & 0.2559 & 0.5911 & 0.4329 \\
\hline SRV & 17.4100 & 12.3900 & 1.4052 & 0.9418 & 2.5206 & 0.3737 \\
\hline SRT & 4.9252 & 2.5542 & 1.9283 & 0.2783 & 0.5786 & 0.4810 \\
\hline T1RT & 1.2481 & 2.3865 & 0.5230 & 0.0401 & 0.6104 & 0.0657 \\
\hline
\end{tabular}


Table 3: Model updating results for the Polymylos and Kavala bridge

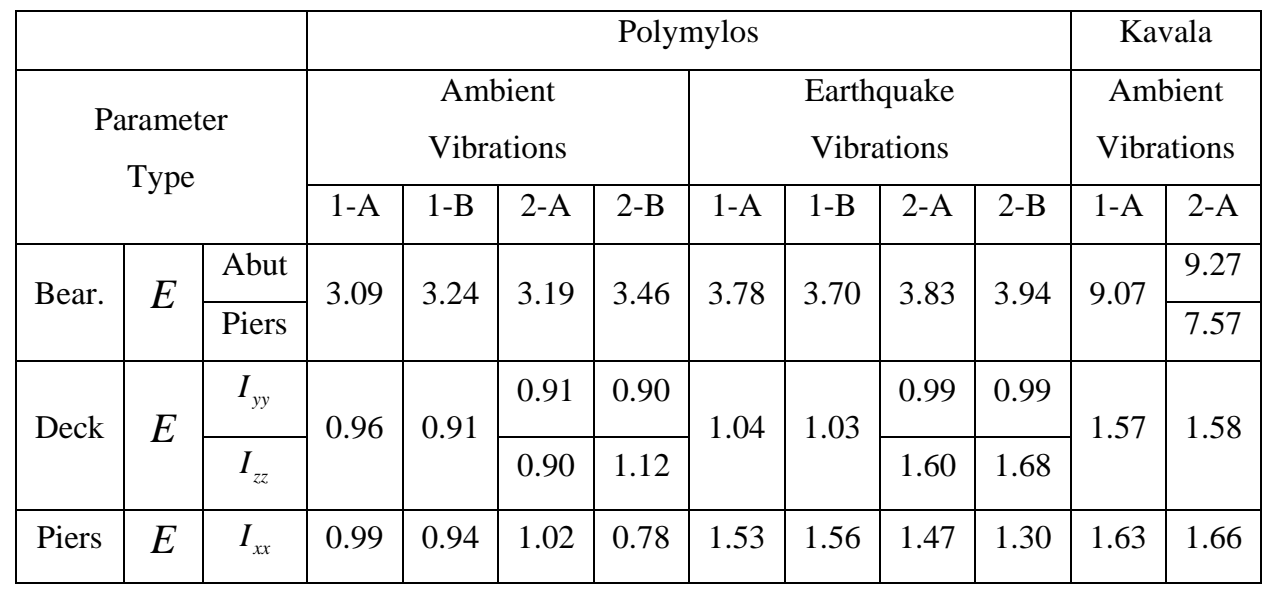


Table 4: Percentage error $\Delta \omega$ between measured and model predicted modal frequencies along with MAC values for the Polymylos bridge

\begin{tabular}{|c|c|c|c|c|c|c|c|c|c|c|c|c|c|c|c|c|}
\hline \multirow[b]{3}{*}{ Mode } & \multicolumn{8}{|c|}{ Ambient Vib } & \multicolumn{8}{|c|}{ Earthquake Vib } \\
\hline & \multicolumn{2}{|c|}{ Case 1-A } & \multicolumn{2}{|c|}{ Case 1-B } & \multicolumn{2}{|c|}{ Case 2-A } & \multicolumn{2}{|c|}{ Case 2-B } & \multicolumn{2}{|c|}{ Case 1-A } & \multicolumn{2}{|c|}{ Case 1-B } & \multicolumn{2}{|c|}{ Case 2-A } & \multicolumn{2}{|c|}{ Case 2-B } \\
\hline & $\begin{array}{c}\Delta \omega \\
\%\end{array}$ & MAC & $\begin{array}{c}\Delta \omega \\
\%\end{array}$ & MAC & $\begin{array}{c}\Delta \omega \\
\%\end{array}$ & MAC & $\begin{array}{c}\Delta \omega \\
\%\end{array}$ & MAC & $\begin{array}{c}\Delta \omega \\
\%\end{array}$ & MAC & $\begin{array}{c}\Delta \omega \\
\%\end{array}$ & MAC & $\begin{array}{c}\Delta \omega \\
\%\end{array}$ & MAC & $\begin{array}{c}\Delta \omega \\
\%\end{array}$ & MAC \\
\hline $1^{\text {st }} \mathrm{T}$ & 3.3 & 0.79 & 3.0 & 0.79 & 4.3 & 0.80 & 0.0 & 0.69 & 1.6 & 0.70 & 1.6 & 0.70 & 2.0 & 0.71 & 0.5 & 0.32 \\
\hline $2^{\text {nd }} T$ & 5.2 & 0.93 & 4.3 & 0.67 & 4.2 & 0.93 & 0.0 & 0.92 & 3.6 & 0.94 & 4.4 & 0.94 & 1.8 & 0.94 & 0.2 & 0.56 \\
\hline $1^{\text {st }} \mathrm{B}$ & 1.4 & 0.98 & 1.2 & 0.88 & 2.3 & 0.99 & 0.0 & 0.99 & 7.6 & 0.99 & 7.6 & 0.99 & 0.6 & 0.99 & 0.6 & 0.99 \\
\hline $3^{\text {rd }} \mathrm{T}$ & 4.0 & 0.76 & 4.7 & 0.51 & 4.5 & 0.77 & 0.0 & 0.76 & 9.3 & 0.72 & 9.7 & 0.72 & 0.5 & 0.73 & 0.4 & 0.73 \\
\hline $2^{\text {nd }} B$ & 3.0 & 0.98 & 0.3 & 0.98 & 0.3 & 0.98 & 0.0 & 0.98 & 3.3 & 0.99 & 2.7 & 0.99 & 0.4 & 0.99 & 0.4 & 0.99 \\
\hline
\end{tabular}

L=Longitudinal, $\mathrm{T}=$ Transverse, $\mathrm{B}=$ Bending (deck) 